\title{
An Efficient Two-Stage User Association Scheme for Green C-RAN Systems
}

\author{
I. Sh. Hburi, H. F. Khazaal
}

Department of Electrical Engineering, Wasit University, Iraq

\begin{tabular}{|c|c|}
\hline Article Info & ABSTRACT \\
\hline Article history: & This paper addresses the energy efficiency (EE) based user-association \\
\hline Received Dec 29, 2018 & $\begin{array}{l}\text { problem in cloud radio-access network. In specific, a low-complexity } 2 \text {-stage } \\
\text { iterative algorithm is developed for this purpose by jointly emploving the }\end{array}$ \\
\hline Revised May1, 2019 & coordinated multi-point (CoMP) and user-clustering techniques. The first \\
\hline Accepted May 26, 2019 & $\begin{array}{l}\text { stage of the algorithm applies a greedy search to form user-cluster and } \\
\text { activate corresponded remote radio heads (RRHs) for CoMP transmission, }\end{array}$ \\
\hline Keywords: & $\begin{array}{l}\text { only an RRH } \mathrm{j} \text { with good channel gain could be included in the serving } \\
\text { cluster of user } \mathrm{k} \text {. Next, in the second stage, a re-clustering procedure is }\end{array}$ \\
\hline Cloud radio access networks & utilized to mitigate inter-cluster interference (ICI) and assign optimal power \\
\hline Coordinated multi-point & $\begin{array}{l}\text { for scheduled users. Our obtained findings confirm that the transmit power, } \\
\text { RRH density, and the number of users served by these RRHs can }\end{array}$ \\
\hline Energy efficiency & significantly affect the EE. Also, the results show that under certain system \\
\hline User scheduling & $\begin{array}{l}\text { settings the proposed scheme can enhance the EE by almost } 39.6 \% \text { compared } \\
\text { to conventional CoMP without user clustering technique. }\end{array}$ \\
\hline
\end{tabular}

Copyright $\odot 2019$ Institute of Advanced Engineering and Science. All rights reserved.

\section{Corresponding Author:}

Ismail Hburi,

Department of Electrical Engineering,

Wasit University,

Al-Rabee District Kut, Wasit, Iraq.

Email: Ismail.hburi@brunel.ac.uk

\section{INTRODUCTION}

Background: Cloud radio-access network (C-RAN) is recognized as an emerging networkarchitecture for fifth generation $(5 \mathrm{G})$ networks that provides significant promises in supporting both the energy efficiency (EE) and the spectrum efficiency (SE). In C-RAN, most of the baseband digital signal processing can be migrated to the cloud-computing cite, refer to, e.g., [1, 2] and the references therein for more details. Thereby, the traditional high-power base stations (BSs) can be replaced by low power radioremote heads (RRHs). Besides, the central processor allows for the coordinated multi point (CoMP) processing for inter-cell interference mitigation. In addition, it can help in joint resource-allocation (RA) among the RRHs to assign resources on demand while putting idle RRHs into sleep-mode for energy-saving (e.g, see references [3, 4]).

The Problem: CoMP technology,on the other hand, comes at the cost of an increase in energy consumption due to the additional power needed to assist the increased backhauling for cooperation particularly with increasing number of users and RRHs. On this basis, for large-scale C-RAN, selecting the serving-RRHs for different user equipment, or referred as user-association, is a key problem. In addition, if all the RRHs are utilised in the transmission then, power consumption for fronthaul links and other circuitry could be very large, which affects the energy efficiency. To this end, accounting for the aforementioned limitation, this work aims to optimise the C-RAN power consumption by jointly optimizing clustering and power allocation problem. But unfortunately, such problem is a high complexity combinatorial problem where the optimal solution needs infeasible exhaustive high-cost search, especially for a large number of users and/or RRHs. 
Related Works: The potential of C-RAN in enhancing the performance of next-generationsystems hasattracted remarkable attention recently. With similar interests as ours, Dongyang et al. in [5] introduce an efficient three-phase algorithm for solving clustering and user association problem. Firstly, based on the lower-bound of the ergodic rate, a greedy association scheme is achieved to create an elementary cluster. And then this cluster is split into many small clusters based on the short-term instantaneous channel information. In [6], Huang et al. present a dynamic association policy to create cluster RRHs and user-groups to mitigate the inter-cell and intra- cell interference. Liu et al. in [7] design a clustering algorithm aimed at optimising the average throughput of C-RAN system subject to the constraint on backhaul-capacity, they consider a hybrid CoMP based a semi-dynamic close to the optimal approach. Alouini et al. in [8] address the joint power control and coordinated association problem in downlink of C-RAN where the metric is maximizing the weighted-sum-rate with some practical constraints.

Ali et al. in [9], consider the joint optimization of remote-radio-heads (RRH) association, sub_channel assignment, and power allocation for network sum_rate maximization in single_carrier frequency division multiple access (SC-FDMA) based multi_tier C-RAN. Ghauch et al. in [10], investigate the issue of mitigating interference between antenna domains of C-RAN system. They formulate the user assignment problem as an integer optimization program, and propose an iterative scheme for the solution. Motivated by the above, this paper addresses the EE based user-association problem in cloud radio-access network, where user-clusters and RRHs-groups are formed for this purpose. Chang et al. in [11] address the user association and resource allocation algorithm for improving EE. They present an optimization formula that jointly addresses user association, subchannel assignment, and power allocation based on the convex optimization tools.

The Proposed Solution: Different from [6], we adopt a 2-stage approach with a hybrid-centric instead of user-centric method in order to make a trade off between cluster edge-users performance and association complexity. In the first stage, clusters are constructed from the users' point-of-view to maximise their signals, while in the second stage users are re-clustered according to the network perspective to mitigate inter-cluster interference. In addition, the analysis considers demand-based irregular-deployment of RRHs rather than the traditional Hexagonal topology. The main contributions of this work can be summarized as follows:

- The problem of energy minimizing in downlink C-RAN system is formulated subject to the total required power and user target SINR. Next, a low complexity two-stage user association scheme is proposed to address issues on high computational cost, high power, and limited fronthaul-capacity.

- A simple closed to optimal power control policy is adopted jointly with RRHs-selection / usersassociation for further improving the EE.

- Performance gain of the proposed scheme with respect to the baselines is deeply investigated and obtained results provide insights into impacts of key design parameters.

\section{RESEARCH METHOD}

The system consist of a C-RAN architecture, as depicted in Figure 1. Let $\mathrm{K}=\{1,2, \cdots, \mathrm{k}\}$ denote the set of single-antenna users deployed within the network and $\mathrm{K}=|\mathcal{K}|$ is the toal number of users. Moreover, the set of RRH nodes are expressed as $\mathcal{J}=\bigcup_{j}\left\{R R H_{\mathrm{j}}\right\}$, with $\mathbf{J}=|\mathcal{J}|$ is the number of available RRHs in the network and RRHj is connected to the BBU-pool via a limited capacity fronthaul link which can be labeled as $\mathrm{Sj}$. Various signal-processing functionalities, e.g. modulation and channel-encoding, are performed at the BBU module and then distributed to the RRH ports through optical fiber Figure 1. Each user is associates its own-cluster within a group of RRHs connected to the same BBU. Muliuser-CoMP is utilized since user useful data is available at all RRHs within the cluster (assuming Ideal fronthaul). Intra-cluster interference is negligible due to Zero-forcing (ZF) strategy employment. k-th user is allocated a cluster of $\mathcal{C}_{j}^{k}$ RRHs with $\left|\mathcal{C}_{J}^{k}\right|=\mathrm{R}$. Total number of the users in the cluster (served on the same time/freq. resources) is $\left|\mathcal{C}_{K}^{k}\right|=\mathrm{U}$ such that a virtual $\mathrm{R} \times \mathrm{U}$ multiple-input multiple-output is created from $\mathcal{C}_{\mathrm{J}}^{\mathrm{k}} \mathrm{RRHs}$ and $\mathcal{C}_{\mathrm{K}}^{\mathrm{k}}$ users. The considered model account precisely for power-consumption of all of these modules. To address issues on high power consumption, high computational complexity, and limited fronthaul-capacity, we utilize an adaptive association scheme to assign a group of RRHs to each user for cooperative transmission.

\subsection{Energy Efficiency Problem Formulation}

To describe the approach used in our scheme, we introduce an RRH-transmission matrix matrix $A \in\{0,1\}^{\mathrm{J} \times \mathrm{K}} 2$ as a binary- matrix with each element of this matrix is either 1 if $\mathrm{j}$ transmits useful data to $\mathrm{UE}_{\mathrm{k}}$ or 0 , otherwise. 


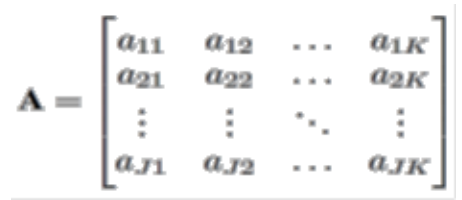

where each non-zero element $\mathrm{a}_{\mathrm{jk}}$ means that the node $\mathrm{RRH}_{\mathrm{j}}$ is involved in current cluster c transmission. De-noting the transmitted and received signals respectively by, $x=\left[x_{1}, x_{2}, \cdots x_{K}\right]^{T}$ and $y=$ $\left[\mathrm{y}_{1}, \mathrm{y}_{2}, \cdots \mathrm{y}_{\mathrm{K}}\right]^{\mathrm{T}}$, wherey $\mathrm{K}_{\mathrm{K}}$ is $\mathrm{UE}_{\mathrm{k}}$ 'sreceived signal and its vector-form can be written as [10]

$$
y=H(A \cdot Q) \sqrt{p} x+z_{n} \cdots
$$

with $\{\mathrm{H}, \mathrm{Q}\} \in \mathbb{C}^{\mathrm{J} \times \mathrm{K}}$ are, respectively, the channel gainand beamforming matrices, (·) means element wise matrix product, $\mathrm{p}$ is the transmission power, and $\mathrm{z}_{\mathrm{n}}$ is an additive-white-Gaussian noise (AWGN) matrix with user element $\boldsymbol{z}_{k} \in C N\left(0, \sigma^{2}\right)$, i.e., a zero mean and a unity variance $\sigma^{2}$ complex-normal distributed random variables (r.vs).

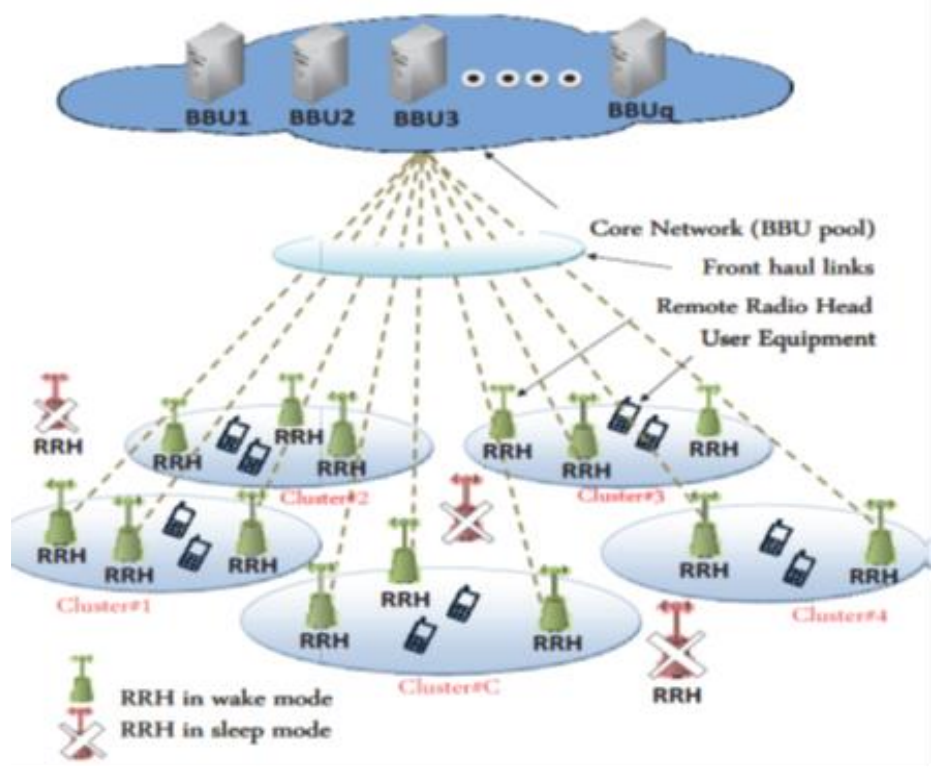

Figure 1. The proposed Cloud-RAN topology, the RRHs are connected to the BBUs Pool via a switch of $10 \mathrm{~Gb} / \mathrm{s}$ Ethernet

Channel coefficients $h_{j k}$ are proposed to be r.vs with independent-and-identically distribution, and they made up of two-terms,fast-fading $g_{j k}$ and shadow-fading coefficients with geometric path loss component $\mu_{j k}, h_{j k}=g_{j k}\left(\mu_{j k}\right)^{1 / 2} . h_{j k}$ is the fast fading component of $g_{j k}$ and the large-scale component canbe expressed as [10], $\mu_{j k}=\beta_{j k} / D_{j k}^{v}$, where, $\beta_{j k}$ is the shadowing r.vs with log-normal-distribution, $D_{j k}$ is the distance from the RRH to the user MUk, and $v$ is the path-loss exponent. With unity average transmitted power, the power expectation ofthe signal is $\Xi\left\{x_{k} \mathrm{x}_{\mathrm{k}}^{\mathrm{H}}\right\}=\Xi\left\{\left|x_{k}\right|^{2}\right\}=1$. Consequently, the resultant signal-tointerference-plus-noise ratio (SINR) at user $U E_{k}$ is, by definition, obtained as

$$
\Gamma_{\mathrm{k}}=\frac{\mathrm{p}_{\mathrm{k}}\left|\sum_{\mathrm{j} \in \phi_{\mathrm{k}}} \Xi\left\{\mathrm{h}_{\mathrm{jk}}^{\mathrm{T}}\left(\mathrm{a}_{\mathrm{jk}} \cdot \mathrm{q}_{\mathrm{jk}}\right)\right\}\right|^{2}}{\sum_{\grave{\mathrm{k}} \in \mathcal{K}, \grave{\mathrm{k}} \neq \mathrm{k}} \mathrm{p}_{\mathrm{k}}\left|\sum_{\mathrm{j} \in \phi_{\grave{k}}} \Xi\left\{\mathrm{h}_{\mathrm{jk}}^{\mathrm{T}}\left(\mathrm{a}_{\mathrm{j} \mathrm{k}} \cdot \mathrm{q}_{\mathrm{j} \mathrm{k}}\right)\right\}\right|^{2}+\mathrm{I}^{\mathrm{IC}}+\frac{\sigma^{2}}{\mathrm{p}_{\mathrm{k}}}}
$$

where $\phi_{k}, \phi_{\mathrm{k}}$ are respectively the RHHs for clusters of users $\mathrm{k}, \mathrm{k}^{\prime}$, the inter-cluster interference (ICI) is defined as 


$$
\mathrm{I}^{\mathrm{IC}}=\sum_{\grave{c} \in C, \grave{c} \neq c} \sum_{\grave{\mathrm{k}} \in \mathcal{K}, \grave{\mathrm{k}} \neq \mathrm{k}} \mathrm{p}_{\mathrm{k}}\left|\sum_{\mathrm{j} \in \phi_{\grave{\mathrm{k}}}} \Xi\left\{\mathrm{h}_{\mathrm{c} \mathrm{j} \mathrm{k}}^{\mathrm{T}}\left(\mathrm{a}_{\mathrm{c} \mathrm{j} \grave{\mathrm{k}}} \cdot \mathrm{q}_{\mathrm{c} \mathrm{j} \mathrm{k}}\right)\right\}\right|^{2}
$$

In order to simplify the notation, cluster index has been omitted from the equation of $\Gamma_{k}$ in (2). The achievable throughput and energy efficiency of cluster $\mathrm{c}$ can be espectively expressed as $[12,13]$

$$
\begin{aligned}
& \mathrm{S}_{\mathrm{c}}=\mathcal{B} \sum_{\mathrm{j} \in \phi_{\mathrm{k}}} \sum_{\mathrm{k} \in \mathcal{K}} \ln \left(1+\operatorname{SINR}_{\mathrm{jk}}\right) \text { (in nats/sec) } \\
& \mathcal{E}_{\mathrm{c}}=\frac{\text { Cluster Throughput }}{\text { Cluster total Power }}=\frac{\sum_{\mathrm{k} \in \mathcal{K}} \mathrm{S}_{\mathrm{k}}}{\mathrm{P}_{\mathrm{k}} \text { total }},
\end{aligned}
$$

where, $\mathcal{B}$ is system Bandwidth, $S_{k}$ is user throughput, and nats is defined as the natural digit $\log _{2}(\mathrm{e}) \approx($ 1.443) bits, with $\mathrm{e}$ is the base of the natural-logarithms. The total power consumption can be expressed as [14]

$$
P^{\text {total }}=\sum_{j \in \phi_{\mathrm{k}}} \frac{Q \mathrm{GP}_{\mathrm{j}} \mathrm{G}^{\mathrm{H}}}{\eta_{\mathrm{j}}}+\mathrm{P}_{\mathrm{j}}^{\mathrm{o}}+\mathrm{P}^{\mathrm{fh}}
$$

with $\eta_{j}$ is the power amplifier efficiency, $\boldsymbol{G}=\boldsymbol{A} . \boldsymbol{Q}$, the static hardware power-consumption is denoted by $P_{j}^{o}, \mathcal{Q}$ is the number of time/frequency resource blocks (RBs), $P^{\mathrm{fh}}$ is the fronthaul-link's power consumption. Therefore, our energy optimisation problem can be expressed as follows

$$
\begin{aligned}
& \max _{\{\mathbf{A}, \mathbf{Q}, \mathbf{P}\}} \sum_{j \in \Phi} \sum_{k \in \mathcal{K}} \mathscr{E}_{j k}^{\text {Cloud-RAN }} \\
& \text { s.t } C_{1}: \quad S_{k} \geq S_{k}^{o}, \forall k \in \mathcal{K} \\
& C_{2}: \quad \mathrm{GP}_{j} \mathrm{G}^{H} \leq P_{j}^{\max }, \forall j \in J \\
& C_{3}: \quad a_{j k} \in\{0,1\}, \quad \forall j \in J \forall k \in \mathcal{K}
\end{aligned}
$$

where $S_{k}^{o}$, in the first per-user constraint of eq. (7a ) represents the minimum downlink data-rate required by user- $k$ to ensure end user's-QoS, the $2^{\text {nd }}$ inequality shows per-RRH constraint to guarantee that not any RRH exceed maximum allowed power $P_{j}^{\max }$. The above efficiency-maximisation formula is a familiar non-convex integer (since $a_{j k}$ is a binary variable) combinatorial, non-polynomial time hard (NP-hard) optimization, so in reality obtaining its exact optimal solution is not easy task especially for systems with large number of served users. Considering high computational-costs of this formula, we invoke a clustering approach to change it into a simple disjoint optimisation algorithm. According to [15], the objective function in eq. (7a) can be expressed as

$$
\mathcal{E}^{*}=\max _{\{\grave{A}, \grave{Q}\}} \frac{\sum_{k \in \mathcal{K}} S_{k}}{P^{\text {total }}}=\frac{S^{o p t}}{P^{o p t}}
$$

where $P^{o p t}$ is the optimal total power consumption, $S^{\text {opt }}$ is the optimum sum-rate, and $\left\{\mathrm{A}^{\prime}, \mathrm{Q}^{\prime}\right\} \in\{\mathrm{A}, \mathrm{Q}\} \mid$ $\{C 1, C 2\}$, i.e, the region of feasibility for the problem of eq. (8). Thus,the optimal efficiency $\mathcal{E}^{*}$ can be achieved if and only-if.

$$
\mathbb{F}(\mathrm{P})=\max _{\{\dot{A}, \hat{Q}\}}\left[\mathrm{S}^{\mathrm{opt}}-\mathrm{P}^{\mathrm{opt}}\right]=0,
$$

Consequently, based on the theoretical results in [16], prob-lem $\mathcal{P}_{1}$ can be transformed as the following parametric-programming formula.

$$
\max _{\{\mathbf{A}, \mathbf{Q}\}} \sum_{k \in \mathcal{K}} S_{k}-P_{c}^{\text {total }}
$$




$$
\text { s.t } C_{1}, C_{2}
$$

It is worth pointing out that if formula $\mathcal{P}_{2}$ is optimally solved such that $\mathbb{F}(\mathbf{P})=0$, then formula $\mathcal{P}_{1}$ can besolved optimally. The expression of eq.(9) is a monotonically-decreasing over power $\mathbf{P}$, i.e., it is a convex problem. Therefore, a bisection approach would perform well enough to obtain the optimal power.

\subsection{Proposed 2-Stage User-Association Algorithm}

As described in the last subsection, the exhausted search has excessively high complexity with increasing number of users and RRHs, which motivates us to devise a practical scheme to solve efficiencyproblem of eq. (7a). Specifically, we offer a low complexity two-stage approach with adaptive power allocation, referred to as Adaptive User aSsociation Algorithm (AUSA), based on non-overlapping or disjoint user-clusters to decouple the main problem into per-cluster subproblems. These optimisation subproblems can be solved at different local clusters simultaneously taking into account the feasibility of the global system requirements (In comparison with disjoint approach, overlapping-clusters need more complex procedure in terms of users' association, network size scalability, and signaling overhead ). For each cluster, users are hosen according to an SINR-threshold such that the created topology surfers a minimum inter-clusterinterference (ICI) with reduced computational-complexity and signaling over-head. The two stages of the AUSA are respectively summarized in Figures 2 and 3. Subsequently, the following discussion elaborates procedures of these two stages.

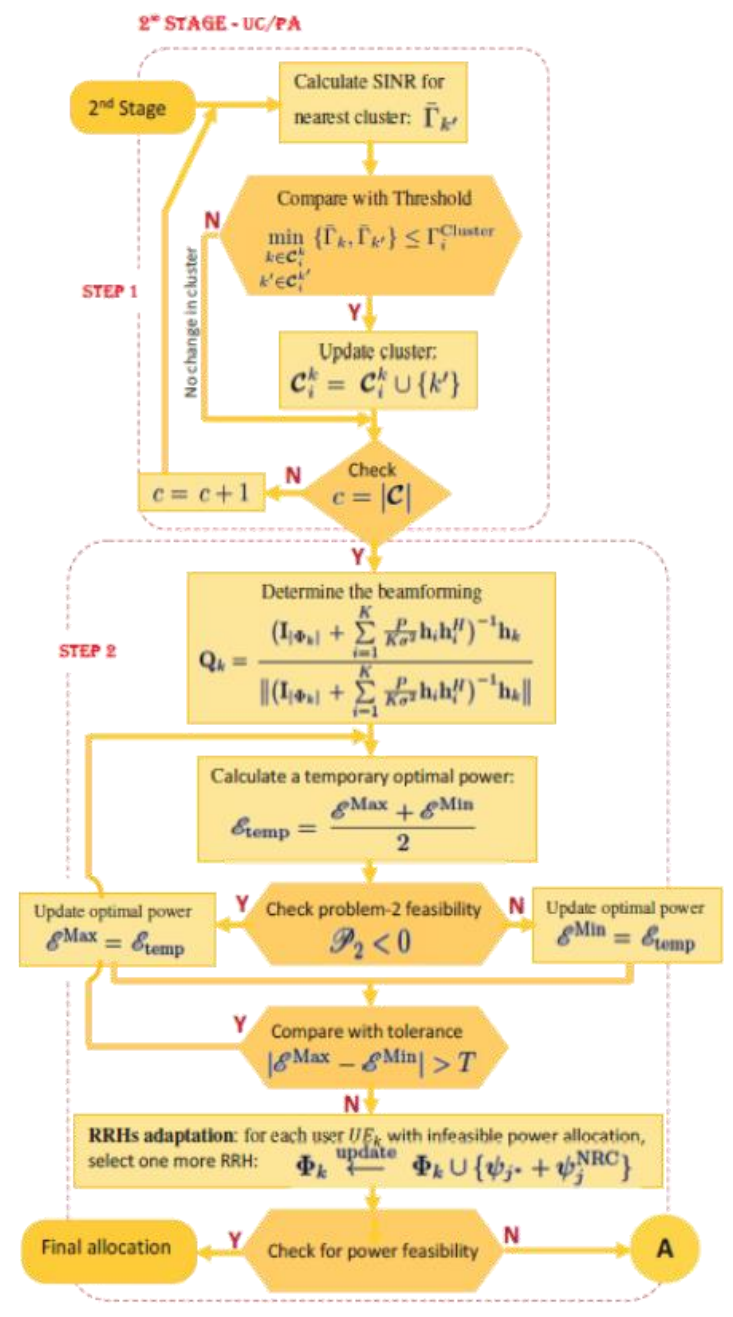

Figure 2. First Stage of the Adaptive User aSsociation Algorithm (AUSA): Construction of RRH-activation matrix (ALPHA)

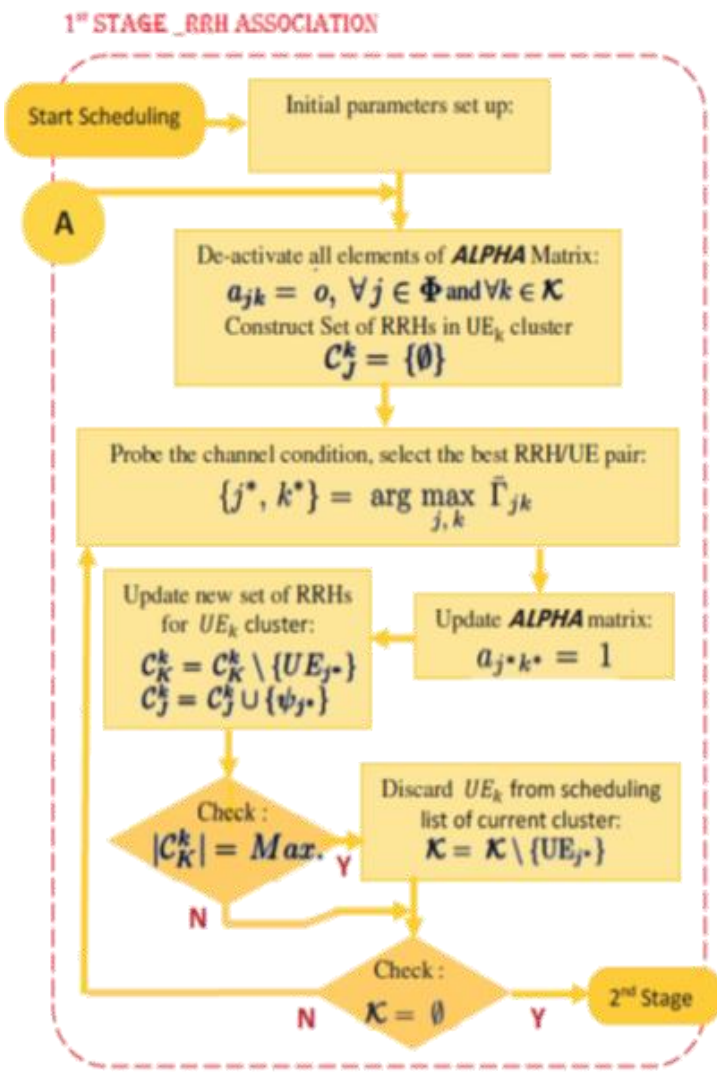

- ALPHAMarix is the RRH selection Matrix

- Average SINR for each link is considered in the decision.

Figure 3. Second Stage of the Adaptive User aSsociation Al-gorithm (AUSA): User Clustering and Power Allocation (UC/PA) strategies in step1 and step2, respectively 
RRHs selection: In the 1st stage Figure 2, after parameters intialisations, iteration procedure starts to determine the elements of the RRHs activation indicator matrix A. Where a greedy-based policy is adopted for this purpose, i.e., RRHs-selection. According to the channel condition, for each user, groups of preference RRHs with best channel-link are quickly and greedily picked out step-by-step at each iteration from the set of unused channel-links for a Coordinated Multi-point Processing (CoMP). This procedure is executed, continually, until all the users are appended to appropriate RRHs. Hence, the network power-consumption can be reduced as the algorithm activates only the RRHs that contribute the CoMP-gains for each user.

User-association and power-allocation: The 2nd stage Figure 3, consists, mainly, of two steps to perform respectively user-association/power-allocation as follows: At the first step, the algorithm compares SINR for the users of nearest-cluster $\Gamma_{k}, k^{\prime} \in C_{i}^{k^{\prime}}$, with that of current one $\overline{\Gamma_{k}}, k^{\prime} \in C_{i}^{k}$. Meanwhile, user will be re-clustered if any one is less than or equal a predefined threshold $\Gamma_{k}$ Cluster. This step is executed, iteratively, for remaining members of the cluster set $\mathrm{C}$. Thereby, as step 1 terminates, users that experience high inter-cluster interference from neighbour clusters will re-cluster into these interfering cluster to be served jointly via zero-forcing multi-user scheme (MU-CoMP). Now, based on the user-association established in the aforementioned procedure, next step introduces multi-user technique (pre-coding method) to schedule users of each cluster simultaneously over the same resource-blocks (RBs). However, instead of pre-coder optimization techniques, in this work a closed-form expression for the beam-forming is considered to achieve a balance between implementation complexity and network performance. As stated in [17], by taking advantages of spatial dgree-of-freedom (DoF) and fronthaul-capacity, the intra-cell interference can be completely suppressed using regularized zero-forcing pre-coder,

$$
Q_{k}=\frac{\left(\mathbf{I}_{\mid \phi_{k}}+\sum_{i=1}^{K} \frac{P}{K \sigma^{2}} h_{k} h_{k}^{H}\right)^{-1} h_{k}}{\left\|\left(\mathbf{I}_{\mid \phi_{k}}+\sum_{i=1}^{K} \frac{P}{K \sigma^{2}} h_{k} h_{k}^{H}\right)^{-1} h_{k}\right\|}
$$

where, $\sigma^{2}$ is the noise-power and $\mathrm{P} / \mathrm{K}$ is the average-transmit-power. This filtering-terminology is equivalent to maximal-ratio transmission (MRT) in noise-limited-scenarios and equivalent to zero-forcing (ZF) in interference-limited-scenarios. According to [17], the operation region of this suboptimal-scheme, from a geometric-perspective, lies in between these two extremes i.e. ZF and MRT. Next, for the power control method, as per our previous discussion, a simple bisection or linear-search policy is adopted to obtain optimal power P*. Also, to further improve network SINR-coverage, for any infeasible RRH/User link-pair, additional Nearest RRH to the Cluster NRC j can be involved in the set of cooperative RRHs _k for this user. Finally, when the network power-consumption stops decreasing, iteration-procedure terminates and outputs the final solution if it feasible. Otherwise, the iteration procedure continues until convergence or maximum number of iterations is reached.

\subsection{Analysis of Computational Complexity}

To serve $K$ users by $|\mathcal{J}|$ RRHsin the traditional optimal User Association Schemes, a central-unit conducts an exhaustive search over all possible RRH-user combinations of which incurs a high time complexity of order $\mathcal{O}\left((|\mathcal{J}| K)^{3.5} .2^{|\mathcal{J}|}\right)$, i.e. exponential relationship with the number of RRHs [18, 19]. On the other hand, for the proposed sheme, the computational-complexity of computing the RZF pre-coder, which arises from the matrix inversion, is of order $\mathcal{O}\left(\mathcal{J} K^{2}\right)$. Also, when bi-section method is adopted to solve power allocation problem, the incurred computational complexity will be $\mathcal{O}\left(|\mathcal{J}| K^{3.5}\right)$ [20]. Therefore, the overall computational-complexity is in the order of $\mathcal{O}\left(|\mathcal{J}| K^{2}+|\mathcal{J}| K^{3.5}\right)$ which is very low compared with exhaust sereach approach.

\section{NUMERICAL RESULTS}

This section presents numerical analysis to evaluate the performance of the proposed association algorithm where the metrics include network energy efficiency (EE) and complementary commutative distribution-function (CCDF) of the users' SINR. The proposed algorithm AUSA is compared with the following three baseline-schemes:

- Heuristic User aSsociation Algorithm (HUSA): User-association and power-allocation are always performed in different time scales. Association is updated in larger-time scale, while power-allocation is adopted to reduce the effect of small-scale fading (scale in msec). Therefore, many researchers only optimise the association with a simple closed- form power control policy instead of the association/power joint optimisation. Particularly, equal power-allocation per each RRH wich is proposed in [21], $P_{R R H}=\frac{P_{c}^{\max }}{\left|C^{k}\right|}$. 
- Tradition User aSsociation Algorithm (TUSA): Many studies, on the other hand, addressed the problem of energy optimising in Cloud-RAN by exploiting full dimension CoMP (using ZF beamforming) rather than partial CoMP. On this basis, ICI will increase since each user is served by all active RRHs on user-centric basis regardless of the network point of view. Thereby, this scheme performs only the RRHs-selection procedure while omits the clustering procedure of stage-2 Figure 3 (single cluster CoMP network).

- Nearest RRH aSsociation Scheme (NRSS): In this common practice and simple scheme, each user is greedily appended to only one RRH with min. distance to the user (no-CoMP) (refer to, e.g., [19] and the references therein). Not that minimum distance can be easily determined from the large- scale fading (LSF) of the specified link.

The simulations adopt a C-RAN down-link scenario with a square coverage area of $1 \mathrm{Km} \times 1 \mathrm{Km}$, where a number of RRHs are randomly placed with Poisson Point Process (PPP) distribution of density $\lambda_{R R H}=\frac{1}{100^{2} \times \pi}$ per $m^{2}$ and users are uniform distributed. Meanwhile, the wireless-channel is modelled as normalized Rayleigh-fading channel followed by the distance-dependent path-loss model, $P L(d)=128.4+36.7 \log _{10}(d)$ in $\mathrm{dB}$, $\mathrm{d}$ is the distance between the RHH and the user in Km. The powerdensity of noise is $-106 \mathrm{dBm} / \mathrm{Hz}$ with the channel-bandwidth ofB $=10 \mathrm{MHz}$. Besides, we set Po $=10 \mathrm{~W}$, $P^{f h}=0.2 W, \eta_{j}=30 \%$ and RRH maximum transmit power Pmax RRH $=20 \mathrm{dBm}(100 \mathrm{~mW})$ if not specified. $8 \times 10^{3}$ independent system realizations are conducted, whereas the channel and locations are randomly generated in each trial.

\subsection{SINR Statistical Distribution}

The Complementary Commutative-Distribution Function (CCDF) of the users' SINR can be interpreted as the probability of users to be in coverage w.r.t a certain predefined threshold or target SINR, $\operatorname{Pr}_{\text {coverage }}=\operatorname{Pr}(S I N R \geq \tau)$. In regard to this metric, it's observed from Figure 4 that the proposed scheme improves SINR-CCDF compared to the three other schemes owing to its features of power and interference controlling. Where the latter feature is the most common cause of users' SINR-outage. The Figure shows, for 50 percentage coverage, that SINR tolerances for AUSA w.r.t HUSA and TUSS are $3 \mathrm{~dB}$ and $8 \mathrm{~dB}$, respectively. In otherwords, with AUSA and 10dB target SINR, unsatisfied users are reduced by $7.1 \%$, $10.2 \%$ and $49.7 \%$ w.r.t HUSA, TUSS, and NRSS, respectively.

\subsection{EE vs. The Number of Users}

Figure 5 shows the effects of the number of served users on the network efficiency in different association techniques. We observe that AUSA algorithm outperforms, remarkably, the reference schemes. Particularly, at the high number of users, where employing CoMP can increase system Spectral Efficiency $(\mathrm{SE})$ at a rate higher than power increment. This, in turn, improves the EE, refer to (4). For instance, with $\mathrm{K}=$ 20, using AUSA technique can grow the EE by $4.5 \%, 8.5 \%$ and $63.5 \%$ when compared to HUSA, TUSS, and NRSS scenarios, respectively. However, the growth rate slows down with higher network load, in specific, when serving users are more than 25 users, where the small density of RRH-deployment w.r.t users can result in a smaller number of user equipment which can benefit from a wider cluster size.

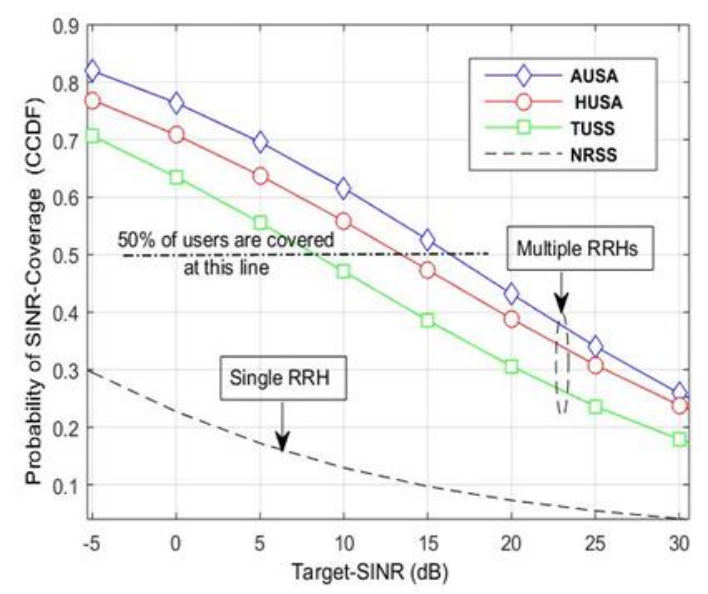

Figure 4. The percentage distribution of user-SINR for $\mathrm{K}=20 \mathrm{users}$ and $\mathrm{P}=20 \mathrm{dBm}$ scenario 


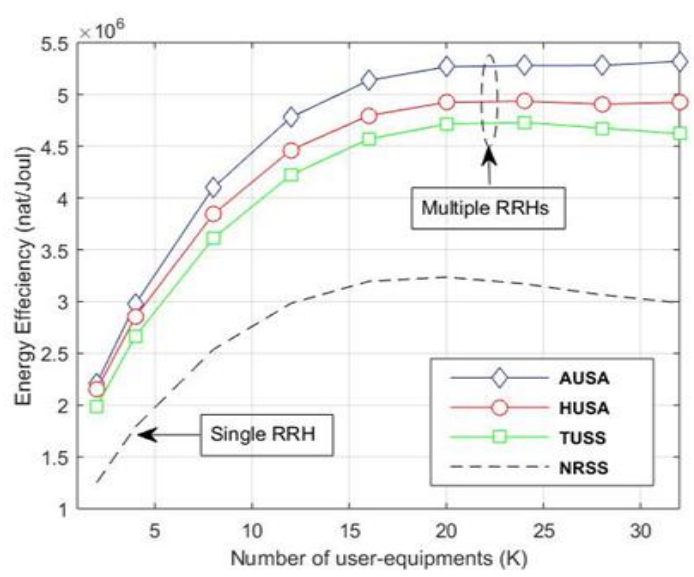

Figure 5. EE vs. K performance for $\lambda \_R R H=1 /\left(100^{\wedge} 2 \times \pi\right)$ [per $\mathrm{m} \rrbracket \wedge 2, \mathrm{P}=20 \mathrm{dBm}$

\subsection{EE vs. RRHs Density}

Figure 6 compares performance of the AUSA association scheme with the baselines schemes (HUSA, TUSS, and RSS) over various RRHs' density. The metric is the Energy Efficiency, where the plot depicts the superior performance of the AUSA scheme for all different number of RRH nodes. For instance, at $\mathrm{J}=20$ RRHs, AUSA enhances the EE by $18.3 \%$, 39.6\%, and 97.4 w.r.t HUSA, TUSS, and NRSS, respectively. The main reason behind the behaviour is that severe distance depending path-loss can be circumvented with the increased number of RRHs. Thereby, RRHs can transmit at lower power level for nearby users, and consequently interference can be reduced. In addition, Figures 7-a and 7-b provide the corresponding EE statistics w.r.t transmit power and RRH-density, respectively. As expected, in Figure 7-a EE decreases with higher transmit power according to (4). On the other hand, Figure 7-b proves that increasing RRH-density beyond a certain value can reduce the efficiency of the network.

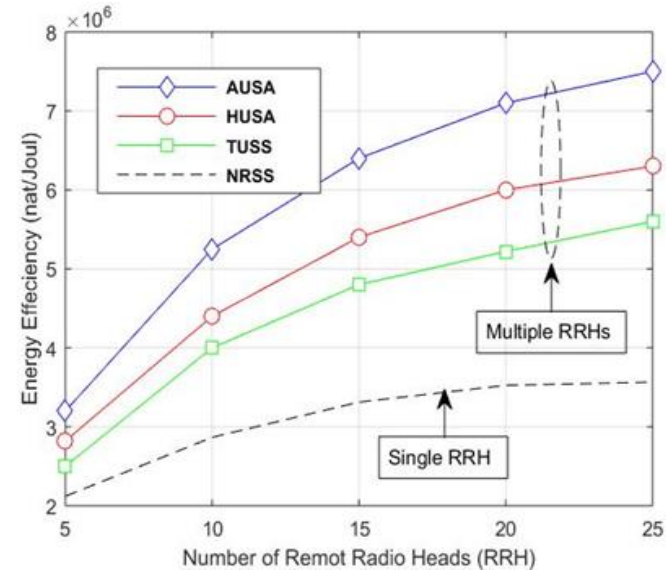

Figure 6. EE vs. RRH for $\mathrm{K}=20$ users and $\mathrm{P}=20 \mathrm{dBm}$
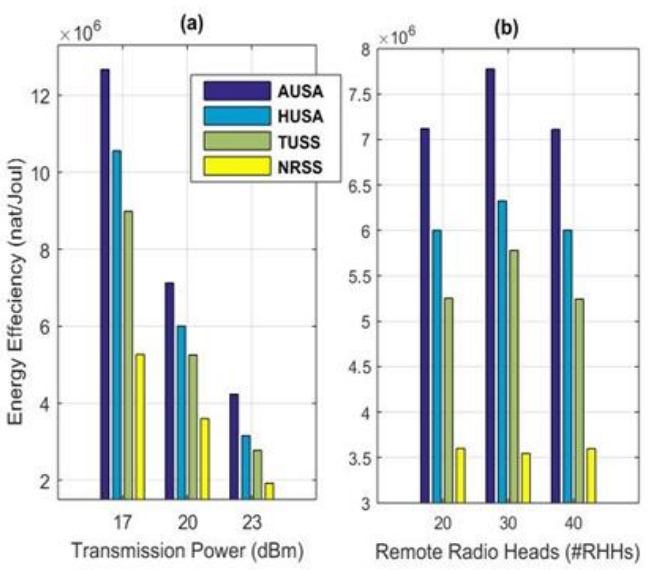

Figure 7. Statistic distribution of EE vs. the power and \#RRH

\section{CONCLUSION}

In this paper, we adopt an energy-saving user association scheme for C-RAN system based on clustering and CoMP transmission. First, a greedy RRH-selection and user-clustering procedures are conducted. Then, a joint interference-aware re-clustering approach and simple power allocation method has been proposed to improve system efficiency under constraints on per-RRH max. power and on per-user min. rate. Numerical results have highlighted the beneficial of RRH-selection and clustering for efficient user association. Besides, the proposed algorithm has superior performance in terms of coverage and scalability with network user number compared to the traditional C-RAN system. 


\section{ACKNOWLEDGEMENTS}

We are thankful to our colleagues in the department of electrical engineering in Wasit University who supported us throughout this work.

\section{REFERENCES}

[1] A. Checko, H. Christiansen, Y. Yan, L. Scolari, G. Kardaras, M. Berger, and L. Dittmann, "Cloud ran for mobile networks-a technology overview," IEEECommun. Surveys \& Tutorials, vol. 17, no. 1, pp. 405-426, 2015.https://ieeexplore.ieee.org/document/6897914.

[2] D. H. Hailu, G. G. lema, S. Bjornstad, "QoS Performance of Integrated Hybrid Optical Network in Mobile Fronthual networks," Indonesian Journal of Electrical Engineering and Computer Science (IJEECS), vol. 7, No. 1, July pp. 189-204. DOI: http://doi.org/10.11591/ijeecs.v7.i1.pp189-204.

[3] D. H. Hailu, G. G. lema, S. Bjornstad, "Performance Evaluation of Ethernet Network for Mobile Fronthual Networks," Indonesian Journal of Electrical Engineering and Computer Science (IJEECS), vol. 7, No. 1, July 2017, pp. 287-298. 2015. DOI: http://doi.org/10.11591/ijeecs.v7.i1.pp287-298.

[4] A. J. Hussein, A. Riadh, M. Alsultan, and A.Tareq, "Applications and Design for a Cloud of Virtual Sensors," Indonesian Journal of Electrical Engineering and Computer Science (IJEECS), vol. 2, No. 1, pp. 229-240, 2016.

[5] X. Dongyang, R. Pinyi, D. Qinghe, and S. Li, "Joint dynamic clustering and user scheduling for downlink cloud radio access network with limited feedback," China Communications, vol. 12(12), pp.147-159. 2015. https://ieeexplore.ieee.org/document/7385522.

[6] X. Huang, G. Xue, R. Yu, and S. Leng, "Joint scheduling and beamforming coordination in cloud radio access networks with QoS guarantees," IEEE Transactions on Vehicular Technology, vol. 65(7), pp.5449-5460. 2016.

[7] D. Liu, S. Han, C. Yang, and Q. Zhang, "Semi-dynamic user-specific clustering for downlink cloud radio access network," IEEE Trans. Veh. Technol., vol. 65, no. 4, pp. 2063-2077, Apr. 2016.

[8] A. Douik, H. Dahrouj, T. Al-Naffouri, and M. Alouini, " Coordinated scheduling and power control in cloud-radio access networks," IEEE Transactions on Wireless Communications, 15(4), pp.2523-2536. 2016.

[9] Sh. Ali, A. Ayaz, I. Razi, S. Sajid, and U. Tariq. "Joint RRH-Association, Sub-Channel Assignment and Power Allocation in Multi-Tier 5G C-Rans." IEEE Accessvol. 6 pp. 34393-34402. 2018. https://ieeexplore.iee.org/document/8387765.

[10] H. Ghauch, M. Rahman, S. Imtiaz, C. Qvarfordt, M. Skoglund, and J. Gross, "User Assignment in C-RAN Systems: Algorithms and Bounds," IEEE Transactions on Wireless Communications, vol. 17(6), pp.3889-3902. 2018.https://arxiv.org/abs/1606.08401.

[11] Z. Chang, J. Gong, T. Ristaniemi, and Z. Niu, "Energy-Efficient Resource Allocation and User Scheduling for Collaborative Mobile Clouds with Hybrid Receivers," IEEE Trans. Vehicular Technology, vol.65(12), pp.9834-9846. 2016.

[12] M. Awais, A. Ahmed, M. Naeem, M. Iqbal, W. Ejaz, A. Anpalagan, and H. Kim, "Efficient joint user association and resource allocation for cloud radio access networks, "IEEE Access, vol. 5, pp.1439-1448. 2017.

[13] I. Hburi and H. Al-Raweshidy, "Outage and average error probability for UL-massive MIMO systems: Asymptotic analysis," IEEE International Conference on Communications (ICC), Paris, pp. 1-6. 2017.https://ieeexplore.ieee.org/document/7996891.

[14] I. Hburi, "Energy efficient cell association in two-tier cellular networks," IEEE International Conference on Advance of Sustainable Engineering and its Application (ICASEA), Wasit, 2018, pp. 79-84. doi:10.1109/ICASEA.2018.8370960.

[15] D. Ng, S. Lo, and R.Schober, "Energy-Efficient Resource Allocation in OFDMA Systems with Large Numbers of Base Station Antennas" IEEE Trans. Wirel. Commun,vol. 11, pp. 3292-3304, 2012.

[16] O. Tervo, L.Tran, and M. Juntti, "Optimal Energy-Efficient Transmit Beamforming for Multi-User MISO Downlink" IEEE Trans. Signal Process, vol. 63, pp. 5574-5587, 2015.

[17] E. Björnson and E. Jorswieck, Optimal Resource Allocation in Coordinated Multi-Cell Systems. Now Publishers, 2013.

[18] J. Tang, W. Tay, and T. Quek, "Cross-layer resource allocation with elastic service scaling in cloud radio access network,"IEEE Transactions on Wireless Communications, vol. 14(9), pp. 5068-5081. 2015.https://ieeexplore.ieee.org/document/7105959.

[19] M. Peng, S. Yan, and H. V. Poor, "Ergodic capacity analysis of remote radio head associations in cloud radio access networks," IEEE Wireless Commun. Lett. vol. 3, no. 4, pp. 365-368, Aug. 2014.

[20] I. Pólik and T. Terlaky, "Interior Point Methods for Nonlinear Optimization. In Nonlinear Optimization,." 1st ed, Springer: Berlin, Germany, 2010.

[21] Z. Shao et al., "Standards-compliant energy-saving schemes for downlink LTE/LTE-advanced networks," in Proc. IEEE PIMRC, Washington, DC, USA, pp. 86-90. Sep. 2014.https://ieeexplore.iee.org/document/7136138. 


\section{BIOGRAPHIES OF AUTHORS}

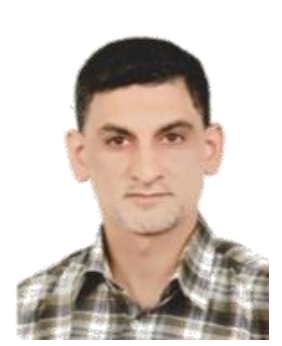

Ismail Sh. B. Hburireceived the B.E. degree in electrical engineering from the University of Technology/Baghdad in 1991, the M.Sc. degree in electrical engineering from the University of Technology/Baghdad in 2007, and the Ph.D. degree in communications and electronics from Brunel University London, U.K., in 2017. He is currently a Lecturer with University of Wasit, Iraq. His experience includes lecturing electronics and communication. His current research area is 5G, C-RAN, IoT, M2M, WSN, and MIMO systems.

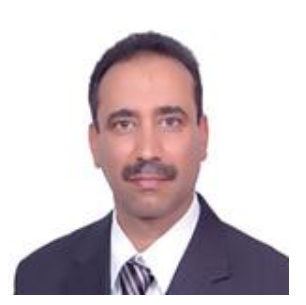

Hasan Al Khazaali,received the B.E. degree in electrical engineering from the University of Technology/Baghdad, the M.Sc. and the Ph.D. degree in electrical engineering from the University of Technology/Baghdad in 2003 and 2010, respectively. He is currently a Lecturer with University of Wasit, Iraq. His experience includes lecturing electronics and communication. His current research area is 5G, Antenna/ waveguide Design, C-RAN, IoT, WSN, and SDR systems. 